\title{
Knowledge Level of Organic and Inorganic Paddy Growers about Cultivation Practices in TBP Command Area
}

\author{
Sidramayya ${ }^{*}$, S. K. Meti ${ }^{1}$, D. M. Chandargi ${ }^{1}$ and Satyanarayana Rao ${ }^{2}$ \\ ${ }^{1}$ Department of Agricultural Extension Education, University of Agricultural Sciences, \\ Raichur, Karnataka, India \\ ${ }^{2}$ Main Agriculture Research Station, University of Agricultural Sciences, \\ Raichur, Karnataka, India \\ *Corresponding author
}

\section{A B S T R A C T}

\section{Keywords}

Knowledge, paddy growers, organic, inorganic and cultivation practices

Article Info

Accepted:

04 November 2019

Available Online:

10 December 2019
Organic farming is gaining popularity all over the world, as it can diversify agricultural production systems towards attaining improved productivity, farm income and food, as well as environmental safety. Hence, the present study analyzes the knowledge level of organic and inorganic paddy growers towards cultivation in TBP Command Area. The major findings indicated that, cent per cent of the organic and inorganic paddy growers had knowledge about recommended varieties (BPT-5204 and Gangavathi sona) for their region as followed by ideal month of sowing in DSR, nursery preparation and manual weed management, while spraying jeevamrutha, use of growth regulators (panchagavya and cow urine), spraying neem oil and cow urine as biological practices of pest and disease management by cent per cent of the organic growers. Whereas, large majority of the organic paddy growers had knowledge about depth of planting $(94.44 \%)$ followed by maintaining 2-3 seedlings per hill $(86.67 \%)$, purpose of summer ploughing as it reduces pest and disease incidence (85.56\%), optimum depth of sowing in DSR (85.56\%), preventing water movement from one field to another (84.44\%), age of seedling (82.22\%) and maintaining $5.0 \mathrm{~cm}$ water level after 10 days of transplanting $(81.11 \%)$. Large majority $(92.22 \%)$ of the inorganic paddy growers had knowledge about depth of planting followed by control measures for brown plant hopper (91.11\%), control measures for stem borer and leaf folder $(87.78 \%)$ and control measures for sucking pests $(84.44 \%)$.

\section{Introduction}

In modern agriculture indiscriminate application of pesticides has resulted in pesticide resistance in insects that compelled to use different molecules and higher dosages. These practices not only increase the cost of product ion but also quality of food is being affected and environment is polluted. A range of alternative eco-friendly methods of pest management practices and for substituting the chemical fertilizers various forms of organic manures and bio-fertilizers are used. Organic agriculture is a unique product ion 
management system which promotes and enhances agro-ecosystem health, including biodiversity, biological cycles and soil biological activities. With the increase in population need not only to stabilize agricultural product ion but to increase it further in sustainable manner. Knowledge has been found to be an important factor contributing to adoption of recommended practices by the farmers. However, the empirical evidences on knowledge possessed on organic and inorganic paddy cultivation practices are much limited. Hence, assessment of organic and inorganic paddy growers knowledge level, towards cultivation practices has become an important issue which needs to be explored. Thus, the present study entitled "A study on knowledge of organic and inorganic paddy growers about paddy cultivation practices in TBP command area".

\section{Materials and Methods}

The present study was conducted in TBP Command Area comprising Raichur, Bellary and Koppal districts during the year 2016-17. These districts were purposively selected because of these districts having more area under paddy cultivation. Two talukas from Raichur and Ballary districts and one taluka from Koppal district were selected based on highest area under paddy. The talukas selected for the study were Sindhanur and Manvi from Raichur district, Siraguppa and Ballary from Ballary district and Gangavathi from Koppal district. Further, all the villages from each taluka who are cultivating paddy under organic condition were selected. Thus the total number of villages selected for the study was thirty eight. From the list prepared a proportionate number of inorganic paddy growers were also selected by using proportionate sampling technique. Under inorganic paddy cultivation it is commonly two methods of cultivation i.e., DSR and transplanting method. Keeping in this mind
180 respondents i.e., 90 transplanting and 90 DSR paddy growers were considerd as respondents in inorganic paddy growers. Thus, making the study sample size 270 comprising of 90 organic paddy growers and 180 inorganic paddy growers.

\section{Results and Discussion}

Knowledge level of organic and inorganic paddy growers about cultivation practices

Overall knowledge level of organic and inorganic paddy growers about cultivation practices

The results presented in Table 1 revealed that, 52.22 per cent of organic paddy growers belonged to medium level of knowledge with respect to organic paddy cultivation practices followed by high and low categories with 37.78 and 10.00 per cent, respectively.

Whereas in case of inorganic paddy growers, a little over half $(53.33 \%)$ of the growers belonged to medium level of knowledge category with respect to transplanting method of paddy cultivation practices followed by high $(28.89 \%)$ and low $(17.78 \%)$ categories.

The probable reasons for this trend (medium to high knowledge level) may be the organic paddy growers and inorganic (transplanting) paddy cultivators in this study area were properly educated, middle to old aged and exposed to the various cultivation techniques used in crop production.

Other probable reasons might be majority of the organic paddy growers participated in various extension activities organized by the NGOs in collaboration with Department of Agriculture might have helped them to gain knowledge. While, in transplanting method, the other factor contributing for medium knowledge may be the farming experience. 
Whereas in case of DSR paddy growers 86.67 per cent of them had high to medium knowledge about paddy cultivation practices. It is evident from the experience that middle aged farmers are more enthusiastic and interested to know more and practice on their field with higher work efficiency than old and young group farmers. The other factors contributing for the above results might be the most of the DSR growers were well educated, more innovative and scientific oriented. The results were in line with results of Sihare (2015).

Knowledge level of organic and inorganic paddy growers about individual cultivation practices

Knowledge level of organic paddy growers about individual cultivation practices

A perusal of Table 2 depicts that among cultivation practices cent per cent of the organic paddy growers had knowledge about recommended varieties (BPT-5204 and Gangavathi sona) for their region as followed by ideal month of sowing in DSR, nursery preparation, spraying jeevamrutha, manual weed management, use of growth regulators (panchagavya and cow urine), spraying neem oil and cow urine as biological practices of pest and disease management.

It was significant observation to note that large majority of the organic paddy growers had knowledge about depth of planting (94.44 $\%$ ) followed by maintaining 2-3 seedlings per hill $(86.67 \%)$, purpose of summer ploughing as it reduces pest and disease incidence $(85.56$ $\%)$, optimum depth of sowing in DSR (85.56 $\%)$, preventing water movement from one field to another (84.44\%), age of seedling $(82.22 \%)$, maintaining $5.0 \mathrm{~cm}$ water level after 10 days of transplanting (81.11\%), recommended spacing (78.89\%), purpose of puddling as it incorporates stubbles (78.89\%), recommended seed rate for transplanting (76.67\%), maintaining $2.5 \mathrm{~cm}$ water level till 10 days of transplanting (76.67\%), purpose of puddling as it control weeds $(75.56 \%)$ and recommended FYM/compost $(70.00 \%)$, respectively.

Since these practices are important aspects of cultivation of any crop, it is necessary for farmers to know about these basic practices perfectly and this might have motivated the farmers to have better knowledge. Another probable reason might be the officials of NGOs in collaboration with Department of Agriculture who are working in the study area might have educated farmers specifically on the above recommended practices.

Over half $(68.89 \%)$ of the organic paddy growers had knowledge about purpose of summer ploughing as it suppresses weed growth followed by intercultivation $(66.67 \%)$, manual with labour as the best method of transplanting (64.44 \%), vermicompost application $(63.33 \%)$, transplanting as the best method for paddy cultivation (62.22\%), use of pheromone trap $(62.22 \%)$, recommended seed rate for DSR $(61.11 \%)$, application of neem cake $(56.67 \%)$, cultivation of green leaf manures $(55.56 \%)$, cultivation of azolla (54.44 \%), and seed treatment with Trichoderma in DSR accounted for 52.22 per cent.

Less than half $(45.56 \%)$ of the organic paddy growers had knowledge about application of poultry manure followed by purpose of summer ploughing as it increases infiltration rate $(43.33 \%)$, DSR as best method for paddy cultivation $(37.78 \%)$, paddy transplanter as best method of transplanting (35.56\%), Pseudomonas fluorescens for blast management $(27.78 \%$ ) and use of Beauveria bassiana for control of stem borer and leaf folder $(26.67 \%)$. An equal per cent $(23.33 \%)$ of the organic growers had knowledge about 
seed dipping in DSR followed by use of cono weeder and use of Metarhizium sp for $\mathrm{BPH}$ control. Whereas, use of Trichograma chilonis for control of stem borer and the purpose of puddling as it conserve water was same attributed to 22.22 per cent and lowest was found in case of seedling treatment for transplanting which were found to be only 16.67 per cent.

The possible reasons for the respondents to be better aware about nutrient and pest management practices might be the fact that, paddy crop suffers from many pests and diseases, so control of these pests and diseases is important for successive cultivation and since it is organic farming no chemical fertilizers are applied and nutrient requirement of crop should be full filled with organic materials only. So, these things might have motivated the farmers to know more about pest and nutrient management practices. Another probable reason might be that, the officials of Department of Agriculture in collaboration with NGOs promoting organic farming technology among farmers through different schemes viz., Savayava Bhagya, Paramparagat Krishi Vikas Yojana, etc. the the results are in conformity with the results of Naik, et al., (2007) and Sihare (2015).

Knowledge level of inorganic paddy growers about transplanting method of paddy cultivation practices

The findings of the Table 2 also reveals that cent per cent of the inorganic paddy growers had knowledge about recommended varieties (BPT-5204 and Gangavathi sona) for their region as every individual tries to acquire knowledge about recommended varieties to get assured yield. Ideal month of sowing in DSR, nursery preparation, application of Zinc sulphate, manual weed management and herbicide application was also found to be cent per cent.
Large majority (92.22\%) of the inorganic paddy growers had knowledge about depth of planting followed by control measures for brown plant hopper (91.11 \%), control measures for stem borer and leaf folder $(87.78$ $\%)$ and control measures for sucking pests $(84.44 \%)$. An equal per cent $(82.22 \%)$ of the growers had knowledge about purpose of summer ploughing as it reduces pest and disease incidence and sheath blight and its management practices. However, number of seedlings per hill, optimum depth of sowing in DSR and age of seedling were accounted for 81.11, 78.89 and 77.78 per cent, respectively. Management practices for blast disease and maintaining water to a depth of $5 \mathrm{~cm}$ for 10 DAT was same attributed to 78.89 per cent. Whereas, recommended seed rate for transplanting and control measures for leaf folder accounted for about 74.44 and 73.33 per cent. An equal per cent (72.22 \%) of the growers had knowledge about recommended spacing and purpose of puddling as it controls weeds. However, purpose of puddling as it incorporates stubbles, purpose of summer ploughing as it suppresses weed growth and maintaining water to a depth of $2.5 \mathrm{~cm}$ for first 10 DAT was same attributed to 70.00 per cent.

These practices are important aspects of cultivation of any crop. Involving low cost and also less complexity of the practices in understanding might be the probable reason for the above findings.

Over half of the inorganic paddy growers had knowledge about manual transplanting and intercultivation was same attributed to 68.89 per cent followed by compost application (63.33\%), recommended seed rate for DSR $(62.22 \%)$, management practices for bacterial leaf blast $(61.11 \%)$, transplanting as best method of paddy cultivation $(56.67 \%)$ and preventing water movement from one field to another $(53.33 \%)$, respectively. Since then paddy is major crop and 51.11 per cent of the 
growers had the farming experience of 9-16 years, followed by 27.17 per cent of the respondents had the farming experience of more than 17 years.

The probable reason for not going for mechanical transplanter is that, special trays and special care is required for rearing paddy seedlings for transplanting in machine, hence the growers go for manual transplanting with labour as there is no such involvements. The results are in line with the results of Sharanappa (2011).

Knowledge level of inorganic paddy growers about DSR method of cultivation practices

The findings of the Table 2 also reveals that cent per cent of the inorganic paddy growers had knowledge about recommended varieties (BPT-5204 and Gangavathi sona) for their region followed by DSR as best method of paddy cultivation, recommended seed rate, ideal month of sowing in DSR, optimum depth of sowing, application of Zinc sulphate, manual weeding, herbicide application, intercultivation and water management practices.

Large majority $(96.67 \%)$ of the inorganic paddy growers had knowledge about management of sheath blight disease followed by control measures for stem borer and leaf folder $(91.11 \%)$, depth of planting $(88.89 \%)$, recommended spacing $(87.78 \%), \quad 2-3$ seedlings per hill $(84.44 \%)$, control measures for Brown plant hopper (83.33\%), 30-35 days old age seedlings $(81.11 \%)$, management of blast $(81.11 \%)$ disease, tractor drawn seed drill is efficient for sowing (78.89 \%), respectively. An equal per cent $(77.78 \%)$ of the growers had knowledge about purpose of summer ploughing as it reduces pest and disease incidence followed by purpose of puddling as it incorporates stubbles and recommended seed rate for transplanting. Manual transplanting as best method of planting and control measures for sucking pests accounted for 76.67 per cent of the growers. 73.33 per cent of them had knowledge about purpose of summer ploughing as it suppresses weed growth.

Over half of the inorganic paddy growers had knowledge about weed management by cycle weeder $(68.89 \%)$ followed by management of bacterial leaf blight disease and purpose of puddling as it controls weeds accounted for 67.78 per cent.

While, 64.44 per cent of the DSR paddy growers had knowledge about alternate wetting and drying of field followed by recommended dose of compost application $(58.89 \%)$ and recommended dose of NPK application (54.44\%). These practices are important aspects of cultivation of any crop.

Involving low cost and also less complexity of the practices in understanding might be the probable reason for the above findings. The findings are in accordance with the studies conducted by Motlhanka et al., (2019).

Knowledge level of organic and inorganic paddy growers about nursery management practices

\section{Knowledge level of organic paddy growers about nursery management practices}

The results presented in the Table 3 conveys that cent per cent of organic paddy growers had knowledge about seeds required for nursery followed by irrigation management, manual weed management in nursery and use of panchagavya as growth regulator. 
Table.1 Overall knowledge level of organic and inorganic paddy growers towards cultivation practices

\begin{tabular}{|c|c|c|c|c|c|c|c|}
\hline \multirow{3}{*}{$\begin{array}{l}\text { Sl. } \\
\text { No. }\end{array}$} & \multirow[t]{3}{*}{ Categories } & \multirow{2}{*}{\multicolumn{2}{|c|}{$\begin{array}{l}\text { Organic paddy } \\
\text { growers }(\mathrm{n} 1=90)\end{array}$}} & \multicolumn{4}{|c|}{ Inorganic paddy growers $(\mathrm{n} 2=180)$} \\
\hline & & & & \multicolumn{2}{|c|}{$\begin{array}{l}\text { Transplanting } \\
(\mathrm{ni}=90)\end{array}$} & \multicolumn{2}{|c|}{$\begin{array}{c}\text { DSR } \\
(\text { nii }=90)\end{array}$} \\
\hline & & $\mathrm{F}$ & $\%$ & $\mathrm{~F}$ & $\%$ & $\mathrm{~F}$ & $\%$ \\
\hline 1 & Low (Mean-0.425*SD) & 9 & 10.00 & 16 & 17.78 & 12 & 13.33 \\
\hline 2 & Medium $($ Mean $\pm 0.425 * \mathrm{SD})$ & 47 & 52.22 & 48 & 53.33 & 36 & 40.00 \\
\hline 3 & High $($ Mean+0.425*SD) & 34 & 37.78 & 26 & 28.89 & 42 & 46.67 \\
\hline & & $\begin{array}{l}\text { Mean: } \\
37.25\end{array}$ & $\begin{array}{l}\text { SD: } \\
4.51\end{array}$ & $\begin{array}{l}\text { Mean: } \\
33.22\end{array}$ & SD: 3.80 & $\begin{array}{l}\text { Mean: } \\
35.54\end{array}$ & $\begin{array}{l}\text { SD: } \\
4.26\end{array}$ \\
\hline
\end{tabular}

Table.2 Knowledge level of organic and inorganic paddy growers about individual cultivation practices

$\mathrm{n}=270$

\begin{tabular}{|c|c|c|c|c|c|c|c|c|}
\hline \multirow{3}{*}{$\begin{array}{l}\text { Sl. } \\
\text { No. }\end{array}$} & \multirow{3}{*}{\multicolumn{2}{|c|}{ Cultivation practices }} & \multirow{2}{*}{\multicolumn{2}{|c|}{$\begin{array}{l}\text { Organic } \\
\text { growers } \\
\left(\mathbf{n}_{1}=90\right)\end{array}$}} & \multicolumn{4}{|c|}{ Inorganic growers $\left(n_{2}=180\right)$} \\
\hline & & & & & \multicolumn{2}{|c|}{$\begin{array}{c}\text { Transplanting } \\
\left(\mathbf{n}_{\mathrm{i}}=90\right)\end{array}$} & \multicolumn{2}{|c|}{$\begin{array}{c}\text { DSR } \\
\left(\mathbf{n}_{\mathrm{ii}}=\mathbf{9 0}\right)\end{array}$} \\
\hline & & & $\mathbf{F}$ & $\%$ & $\mathbf{F}$ & $\%$ & $\mathbf{F}$ & $\%$ \\
\hline $\mathbf{1}$ & \multicolumn{8}{|c|}{ Land preparation } \\
\hline $\mathbf{i}$ & \multicolumn{2}{|c|}{ Purpose of summer ploughing* } & & & & & & \\
\hline & \multicolumn{2}{|c|}{ Increases infiltration rate } & 39 & 43.33 & 36 & 40.00 & 32 & 35.56 \\
\hline & \multicolumn{2}{|c|}{ Suppresses weed growth } & 62 & 68.89 & 63 & 70.00 & 66 & 73.33 \\
\hline & \multicolumn{2}{|c|}{ Reduces pest and diseases incidence } & 77 & 85.56 & 74 & 82.22 & 70 & 77.78 \\
\hline \multirow[t]{4}{*}{ ii } & \multicolumn{2}{|c|}{ Purpose of puddling* } & & & & & & \\
\hline & & 68 & 75.56 & 65 & 72.22 & 61 & 67.78 \\
\hline & \multicolumn{2}{|c|}{ Conserve water } & 20 & 22.22 & 18 & 20.00 & 32 & 35.56 \\
\hline & \multicolumn{2}{|c|}{ Incorporation of stubbles } & 71 & 78.89 & 63 & 70.00 & 70 & 77.78 \\
\hline \multirow[t]{2}{*}{2} & \multirow{2}{*}{$\begin{array}{l}\text { Recommended } \\
\text { varieties }\end{array}$} & BPT-5204 & 90 & 100.0 & 90 & 100.0 & 90 & 100.0 \\
\hline & & Gangavathi sona & 90 & 100.0 & 90 & 100.0 & 90 & 100.0 \\
\hline \multirow[t]{2}{*}{3} & \multirow{2}{*}{$\begin{array}{l}\text { Best method for } \\
\text { paddy cultivation }\end{array}$} & Transplanting & 56 & 62.22 & 51 & 56.67 & 27 & 30.00 \\
\hline & & DSR & 34 & 37.78 & 39 & 43.33 & 90 & 100.0 \\
\hline \multirow[t]{2}{*}{4} & \multirow{2}{*}{$\begin{array}{c}\text { Recommended } \\
\text { seed rate }(\mathrm{kg} / \mathrm{ac}) *\end{array}$} & Transplanting: $25 \mathrm{~kg}$ & 69 & 76.67 & 67 & 74.44 & 70 & 77.78 \\
\hline & & DSR : $8-12 \mathrm{~kg}$ & 55 & 61.11 & 56 & 62.22 & 90 & 100.0 \\
\hline 5 & \multicolumn{2}{|c|}{ Ideal month of sowing in DSR: July } & 90 & 100.0 & 90 & 100.0 & 90 & 100.0 \\
\hline 6 & \multicolumn{2}{|c|}{ Nursery preparation } & 90 & 100.0 & 90 & 100.0 & 90 & 100.0 \\
\hline 7 & \multicolumn{8}{|c|}{ Seed /seedling treatment* } \\
\hline $\mathbf{i}$ & \multicolumn{2}{|c|}{$\begin{array}{l}\text { Seed dipping with Pseudomonas } \\
\text { fluorescens @ } 10 \mathrm{~g} / \mathrm{lt}(30 \mathrm{~min})\end{array}$} & 21 & 23.33 & $* *$ & $* *$ & $* *$ & $* *$ \\
\hline ii & \multicolumn{2}{|c|}{$\begin{array}{l}\text { Seed treatment with Trichoderma sps.@ } \\
4 \mathrm{gm} / \mathrm{kg} \text { of seed }\end{array}$} & 47 & 52.22 & 8 & 8.89 & 0 & 0.0 \\
\hline iii & \multicolumn{2}{|c|}{$\begin{array}{l}\text { Seedling treatment with Azospirillum } \\
\text { sps.@200 g + PSB @ 200g + } \\
\text { Trichoderma sps. @ } 400 \mathrm{~g} / \mathrm{ac}\end{array}$} & 15 & 16.67 & $* *$ & $* *$ & $* *$ & $* *$ \\
\hline
\end{tabular}




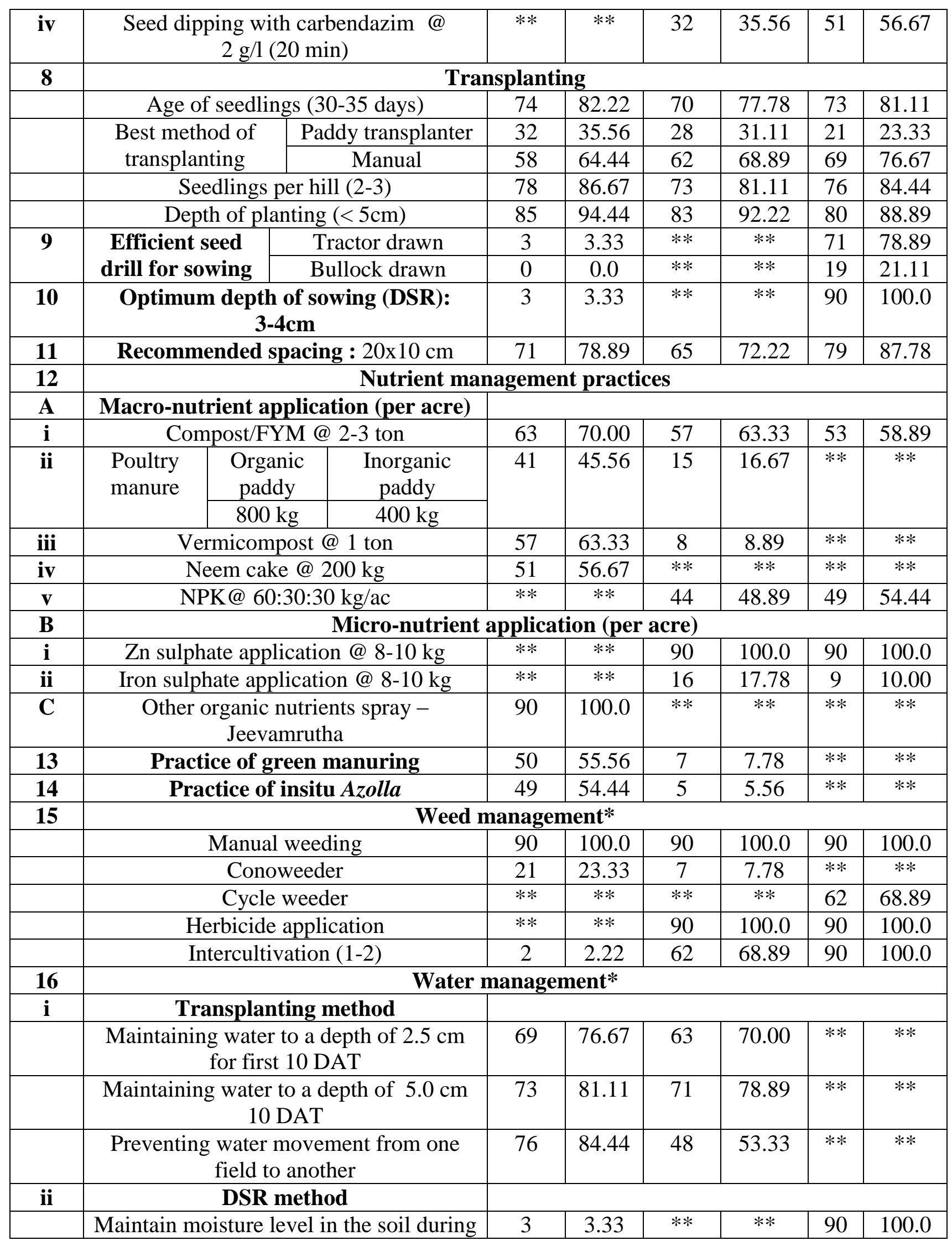




\begin{tabular}{|c|c|c|c|c|c|c|c|c|}
\hline & \multicolumn{2}{|c|}{ early stage before start of irrigation } & & & & & & \\
\hline & \multicolumn{2}{|c|}{ Alternate wetting and drying } & 3 & 3.33 & $* *$ & $* *$ & 58 & 64.44 \\
\hline \multirow[t]{2}{*}{17} & \multirow{2}{*}{$\begin{array}{l}\text { Use of growth } \\
\text { regulators* }\end{array}$} & Panchagavya@3\% & 90 & 100.0 & $* *$ & $* *$ & $* *$ & $* *$ \\
\hline & & Cow urine @ 20\% & 90 & 100.0 & $* *$ & $* *$ & $* *$ & $* *$ \\
\hline 18 & \multicolumn{8}{|c|}{ Plant protection measures } \\
\hline $\mathbf{i}$ & Cultural p & ractices: Summer ploughing & 90 & 100.0 & 90 & 100.0 & 90 & 100.0 \\
\hline \multirow[t]{2}{*}{ ii } & \multicolumn{2}{|c|}{ Mechanical practices } & & & & & & \\
\hline & \multicolumn{2}{|c|}{ Pheromone traps (2/ac) } & 56 & 62.22 & $* *$ & $* *$ & $* *$ & $* *$ \\
\hline \multirow[t]{8}{*}{ iii } & \multicolumn{8}{|c|}{ Biological practices } \\
\hline & \multicolumn{2}{|r|}{ Biofungicide } & & & & & & \\
\hline & \multicolumn{2}{|c|}{$\begin{array}{c}\text { Metarhiziumsp - Brown plant hopper } \\
(\mathrm{BPH})\end{array}$} & 21 & 23.33 & $* *$ & $* *$ & $* *$ & $* *$ \\
\hline & \multicolumn{2}{|c|}{$\begin{array}{l}\text { Beauveriabassiana- Stem borer and leaf } \\
\text { folder }\end{array}$} & 24 & 26.67 & $* *$ & $* *$ & $* *$ & $* *$ \\
\hline & \multicolumn{2}{|c|}{$\begin{array}{c}\text { Biobactericide: Pseudomonas } \\
\text { fluorescens - Blast }\end{array}$} & 25 & 27.78 & $* *$ & $* *$ & $* *$ & $* *$ \\
\hline & \multicolumn{2}{|c|}{$\begin{array}{c}\text { Predator usage: } \text { Trichograma chilonis - } \\
\text { Stem borer }\end{array}$} & 20 & 22.22 & $* *$ & $* *$ & $* *$ & $* *$ \\
\hline & \multicolumn{2}{|c|}{ Neem oil - Sucking pest, stem borer } & 90 & 100.0 & $* *$ & $* *$ & $* *$ & $* *$ \\
\hline & \multicolumn{2}{|c|}{ Cow urine - Bacterial leaf blight } & 90 & 100.0 & $* *$ & $* *$ & $* *$ & $* *$ \\
\hline iv & \multicolumn{2}{|r|}{ Chemical practices } & & & & & & \\
\hline \multirow[t]{4}{*}{$\mathbf{a}$} & $\begin{array}{l}\text { Insect } \\
\text { pests }\end{array}$ & Control measures & & & & & & \\
\hline & $\begin{array}{l}\text { Sucking } \\
\text { pests }\end{array}$ & $\begin{array}{c}\text { Phorate 10G @75g } \\
\text { Monocrotophos@1.3 ml/1 }\end{array}$ & $* *$ & $* *$ & 76 & 84.4 & 69 & 76.67 \\
\hline & $\begin{array}{c}\text { Stem } \\
\text { borer and } \\
\text { leaf folder }\end{array}$ & $\begin{array}{c}\text { Fipronil @ } 2 \mathrm{ml} / 1 \text { or } \\
\text { Chloropyriphos @ } 2 \mathrm{ml} / 1 \text { or } \\
\text { Carbofuran @ } 7.6 \mathrm{~kg} / \mathrm{ac} \\
\text { Profenophos @ } 2 \mathrm{ml} / \mathrm{l}\end{array}$ & $* *$ & $* *$ & 79 & 87.78 & 82 & 91.11 \\
\hline & $\begin{array}{l}\text { Brown } \\
\text { plant } \\
\text { hopper }\end{array}$ & $\begin{array}{l}\text { Buprofezin @ } 1.5 \mathrm{ml} / 1 \text { or } \\
\text { Flonicamid @ } 0.2 \mathrm{~g} / \mathrm{lt}\end{array}$ & $* *$ & $* *$ & 82 & 91.11 & 75 & 83.33 \\
\hline \multirow[t]{4}{*}{$\mathbf{b}$} & Diseases & Management practices & & & & & & \\
\hline & Blast & $\begin{array}{c}\text { Tricyclazole @0.6 g/ 1 or } \\
\text { Cardendenzim@1 g/ } 1\end{array}$ & $* *$ & $* *$ & 71 & 78.89 & 73 & 81.11 \\
\hline & $\begin{array}{l}\text { Sheath } \\
\text { blight }\end{array}$ & $\begin{array}{c}\text { Hexaconazole @ } 1.5 \mathrm{ml} / \mathrm{l} \\
\text { or Propiconazole @ } 1 \mathrm{ml} / \mathrm{l}\end{array}$ & $* *$ & $* *$ & 74 & 82.22 & 87 & 96.67 \\
\hline & $\begin{array}{c}\text { Bacterial } \\
\text { leaf blight }\end{array}$ & $\begin{array}{c}\text { COC @ } 0.12 \mathrm{~g} / 1+ \\
\text { Agrimycin @ } 0.3 \mathrm{~g} / 1\end{array}$ & $* *$ & $* *$ & 55 & 61.11 & 61 & 67.78 \\
\hline
\end{tabular}


Table.3 Knowledge level of organic and inorganic paddy growers regarding nursery management practices

\begin{tabular}{|c|c|c|c|c|c|c|}
\hline \multirow[t]{2}{*}{$\begin{array}{l}\text { Sl. } \\
\text { No. }\end{array}$} & \multirow{2}{*}{\multicolumn{2}{|c|}{ Nursery management practices }} & \multicolumn{2}{|c|}{$\begin{array}{l}\text { Organic paddy } \\
\text { growers }\left(n_{1}=90\right)\end{array}$} & \multicolumn{2}{|c|}{$\begin{array}{l}\text { Inorganic paddy } \\
\text { growers }\left(\mathbf{n}_{2}=90\right)\end{array}$} \\
\hline & & & $\mathbf{F}$ & $\%$ & $\mathbf{F}$ & $\%$ \\
\hline 1 & $\begin{array}{r}\text { Nursery area } \\
\text { ac }\end{array}$ & $\begin{array}{l}\text { I for planting one } \\
\text { qmt) }\end{array}$ & 0 & 0.0 & 0 & 0.0 \\
\hline 2 & Seeds $r$ & $(18-25 \mathrm{~kg})$ & 90 & 100.00 & 90 & 100.00 \\
\hline 3 & $\begin{array}{r}\text { Seeds soaking i } \\
48 \mathrm{hr}\end{array}$ & $\begin{array}{l}(24 \text { hrs) than kept } \\
\text { mination }\end{array}$ & 59 & 65.56 & 38 & 42.22 \\
\hline \multirow[t]{5}{*}{4} & \multicolumn{2}{|c|}{ Fertilizer application } & & & & \\
\hline & \multicolumn{2}{|c|}{ Compost@750 Kg } & 43 & 47.78 & 21 & 23.33 \\
\hline & \multicolumn{2}{|c|}{ Time of application (during puddling) } & 58 & 64.44 & 39 & 43.33 \\
\hline & \multicolumn{2}{|c|}{ NPK@3:1.2:1.5 kg } & $* *$ & $* *$ & 28 & 31.11 \\
\hline & \multicolumn{2}{|c|}{ Time of application (15 \& 30 DAS) } & $* *$ & $* *$ & 72 & 80.00 \\
\hline \multirow[t]{3}{*}{5} & \multicolumn{2}{|c|}{ Irrigation management } & & & & \\
\hline & \multicolumn{2}{|c|}{$\begin{array}{c}\text { Care should be taken not to allow the } \\
\text { nursery to dry for some days }\end{array}$} & 90 & 100.0 & 90 & 100.0 \\
\hline & \multicolumn{2}{|c|}{$\begin{array}{l}\text { Light irrigation is to be done when plants } \\
\text { attain a height of } 1-2 \text { inch }\end{array}$} & 90 & 100.0 & 90 & 100.0 \\
\hline \multirow[t]{4}{*}{6} & \multicolumn{2}{|c|}{ Weed management* } & & & & \\
\hline & \multicolumn{2}{|c|}{ Manual } & 90 & 100.0 & 90 & 100.0 \\
\hline & \multirow{2}{*}{$\begin{array}{l}\text { Herbicide } \\
\text { application }\end{array}$} & Pre-emergent & $* *$ & $* *$ & 90 & 100.0 \\
\hline & & Post-emergent & $* *$ & $* *$ & 90 & 100.0 \\
\hline \multirow[t]{2}{*}{7} & \multicolumn{2}{|c|}{ Use of growth regulator } & & & & \\
\hline & \multicolumn{2}{|c|}{ Panchagavya@3\% } & 90 & 100.0 & $* *$ & $* *$ \\
\hline \multirow[t]{4}{*}{8} & \multicolumn{2}{|c|}{ Important pest and diseases } & & & & \\
\hline & \multicolumn{2}{|c|}{ Important insects: Sucking pests } & 49 & 54.44 & 68 & 75.56 \\
\hline & \multicolumn{2}{|c|}{ Important diseases : Damping off } & 25 & 27.78 & 46 & 51.11 \\
\hline & \multicolumn{2}{|c|}{ Reddening } & 37 & 41.11 & 59 & 65.56 \\
\hline
\end{tabular}


Table.4 Knowledge level of organic and inorganic paddy growers regarding nutrient management practices in main field

\begin{tabular}{|c|c|c|c|c|c|c|c|c|}
\hline \multirow{3}{*}{$\begin{array}{l}\text { Sl. } \\
\text { No. }\end{array}$} & \multirow{3}{*}{\multicolumn{2}{|c|}{ Sources of nutrients }} & \multirow{2}{*}{\multicolumn{2}{|c|}{$\begin{array}{c}\text { Organic } \\
\text { growers } \\
\left(n_{1}=90\right)\end{array}$}} & \multicolumn{4}{|c|}{ Inorganic growers $\left(\mathrm{n}_{2}=\mathbf{1 8 0}\right)$} \\
\hline & & & & & \multicolumn{2}{|c|}{$\begin{array}{c}\text { Transplanting } \\
\left(\mathbf{n}_{\mathrm{i}}=90\right)\end{array}$} & \multicolumn{2}{|c|}{$\begin{array}{c}\text { DSR } \\
\left(\mathbf{n}_{\mathrm{ii}}=90\right)\end{array}$} \\
\hline & & & $\mathbf{F}$ & $\%$ & $\mathbf{F}$ & $\%$ & $\mathbf{F}$ & $\%$ \\
\hline I. & \multicolumn{8}{|c|}{ Organic sources } \\
\hline $\mathbf{A}$ & \multicolumn{2}{|c|}{ Bulky solid sources } & & & & & & \\
\hline $\mathbf{1}$ & \multicolumn{2}{|c|}{ FYM/ compost @ 2-3 ton/ac } & 66 & 73.33 & 57 & 63.33 & 60 & 66.67 \\
\hline 2 & \multicolumn{2}{|c|}{ Poultry manure @ 0.8-1.0 ton/ac } & 31 & 34.44 & 6 & 6.67 & $* *$ & $* *$ \\
\hline & \multicolumn{2}{|c|}{ Or } & & & & & & \\
\hline 3 & \multicolumn{2}{|c|}{ Vermicompost @ 1.0 ton/ac } & 57 & 63.33 & 8 & 8.89 & $* *$ & $* *$ \\
\hline 4 & \multicolumn{2}{|c|}{ Neem cake @ 200 kg/ac* } & 51 & 56.67 & $* *$ & $* *$ & $* *$ & $* *$ \\
\hline \multirow[t]{2}{*}{$\mathbf{i}$} & \multicolumn{2}{|c|}{ Quantity: $100 \mathrm{~kg} / \mathrm{ac}$} & 44 & 48.89 & $* *$ & $* *$ & $* *$ & $* *$ \\
\hline & \multicolumn{2}{|c|}{ Time of application: $30-35$ DAT } & 52 & 57.78 & $* *$ & $* *$ & $* *$ & $* *$ \\
\hline \multirow[t]{5}{*}{ ii } & \multicolumn{2}{|c|}{ Quantity : $100 \mathrm{~kg} / \mathrm{ac}$} & 58 & 64.44 & $* *$ & $* *$ & $* *$ & $* *$ \\
\hline & \multicolumn{2}{|c|}{ Time of application: $55-60$ DAT } & 63 & 70.00 & $* *$ & $* *$ & $* *$ & $* *$ \\
\hline & \multicolumn{2}{|c|}{ Purpose of application:* } & & & & & & \\
\hline & \multicolumn{2}{|c|}{ Insect pest management } & 62 & 68.89 & $* *$ & $* *$ & $* *$ & $* *$ \\
\hline & \multicolumn{2}{|c|}{ Acts as source of nutrients } & 47 & 52.22 & $* *$ & $* *$ & $* *$ & $* *$ \\
\hline 5 & \multicolumn{2}{|c|}{ Cultivation of green leaf manures } & 50 & 55.56 & 7 & 7.78 & & \\
\hline \multirow[t]{4}{*}{$\mathbf{i}$} & \multirow{3}{*}{$\begin{array}{c}\text { Green manure } \\
\text { crops* }\end{array}$} & Dhaincha & 47 & 52.22 & 0 & 0.0 & $* *$ & $* *$ \\
\hline & & Sunhemp & 55 & 61.11 & 4 & 4.44 & $* *$ & $* *$ \\
\hline & & Pillipesaru & 64 & 71.11 & 9 & 10.00 & $* *$ & $* *$ \\
\hline & Seed rate: & $-12 \mathrm{~kg} / \mathrm{ac}$ & 45 & 50.00 & 0 & 0.0 & $* *$ & $* *$ \\
\hline ii & Green leaf & Gliricidia & 40 & 44.44 & 0 & 0.0 & $* *$ & $* *$ \\
\hline & manure crops* & $\begin{array}{c}\text { Leucaenasp } \\
\text { (subabul) }\end{array}$ & 46 & 51.11 & 0 & 0.0 & $* *$ & $* *$ \\
\hline & Application $\mathrm{q}$ & tity: 4 ton/ac & 21 & 23.33 & 0 & 0.0 & $* *$ & $* *$ \\
\hline 6 & Cultivati & of Azolla & 49 & 54.44 & 5 & 5.56 & $* *$ & $* *$ \\
\hline & Quantity req & d: $25 \mathrm{~kg} / \mathrm{ac}$ & 26 & 28.89 & 0 & 0.0 & $* *$ & $* *$ \\
\hline B & & Non-l & y lic & source & & & & \\
\hline 1 & Jeeva & utha & 90 & 100.0 & $* *$ & $* *$ & $* *$ & $* *$ \\
\hline & Method of & eparation & 65 & 72.22 & $* *$ & $* *$ & $* *$ & $* *$ \\
\hline & Quantity to b & orayed: $10 \%$ & 65 & 72.22 & $* *$ & $* *$ & $* *$ & $* *$ \\
\hline & Purpose & praying: & & & & & & \\
\hline & Boost pl & growth & 63 & 70.00 & $* *$ & $* *$ & $* *$ & $* *$ \\
\hline 2 & Panch & javya & 90 & 100.0 & $* *$ & $* *$ & $* *$ & $* *$ \\
\hline & Method of & eparation & 67 & 74.44 & $* *$ & $* *$ & $* *$ & $* *$ \\
\hline & Quantity to & prayed: $3 \%$ & 67 & 74.44 & $* *$ & $* *$ & $* *$ & $* *$ \\
\hline & Purpose o & raying:* & & & & & & \\
\hline & Growth & omoter & 54 & 60.00 & $* *$ & $* *$ & $* *$ & $* *$ \\
\hline & Increa & yield & 46 & 51.11 & $* *$ & $* *$ & $* *$ & $* *$ \\
\hline & Provide immun & in plant system & 11 & 12.22 & $* *$ & $* *$ & $* *$ & $* *$ \\
\hline
\end{tabular}




\begin{tabular}{|c|c|c|c|c|c|c|c|}
\hline $\mathbf{3}$ & Cow urine & 90 & 100.0 & $* *$ & $* *$ & $* *$ & $* *$ \\
\hline & Quantity to be sprayed: 20\% & 52 & 57.78 & $* *$ & $* *$ & $* *$ & $* *$ \\
\hline & Purpose of spraying:* & \multicolumn{7}{|l|}{} \\
\hline & Serve as the urea supplement & 43 & 47.78 & $* *$ & $* *$ & $* *$ & $* *$ \\
\hline & Insecticidal property & 31 & 34.44 & $* *$ & $* *$ & $* *$ & $* *$ \\
\hline II & Inorganic sources & & & & \\
\hline A & Macro-nutrients & $* *$ & $* *$ & 38 & 42.22 & 52 & 57.78 \\
\hline $\mathbf{1}$ & Nitrogen @ 60 kg /ac & $* *$ & $* *$ & 90 & 100.0 & 90 & 100.0 \\
\hline & Split application (top dressing) & $* *$ & $* *$ & 35 & 38.89 & 47 & 52.22 \\
\hline $\mathbf{2}$ & Phosphorus @ 30 kg /ac & $* *$ & $* *$ & 29 & 32.22 & 41 & 45.56 \\
\hline $\mathbf{3}$ & Potash @ 30 kg /ac & & & & \\
\hline B & Micro-nutrients & $* *$ & $* *$ & 90 & 100.0 & 90 & 100.0 \\
\hline $\mathbf{1}$ & Zinc sulphate @ 8-10 kg /ac & $* *$ & 16 & 17.78 & 21 & 23.33 \\
\hline $\mathbf{2}$ & Iron sulphate @ 8-10 kg /ac & $* *$ & $* *$ &
\end{tabular}

*-Multiple responses obtained $\quad * *$-Practices not recommended

Table.5 Knowledge on traditional practices of organic and inorganic paddy growers about plant protection measures

\begin{tabular}{|c|c|c|c|c|c|c|c|}
\hline \multirow{3}{*}{$\begin{array}{l}\text { Sl. } \\
\text { No. }\end{array}$} & \multirow[t]{3}{*}{ Plant protection measures } & \multirow{2}{*}{\multicolumn{2}{|c|}{$\begin{array}{l}\text { Organic paddy } \\
\text { growers }\left(n_{1}=90\right)\end{array}$}} & \multicolumn{4}{|c|}{ Inorganic paddy growers $\left(n_{2}=180\right)$} \\
\hline & & & & \multicolumn{2}{|c|}{$\begin{array}{c}\text { Transplanting } \\
\left(\mathrm{n}_{\mathrm{i}}=90\right)\end{array}$} & \multicolumn{2}{|c|}{$\begin{array}{c}\text { DSR } \\
\left(\mathbf{n}_{\mathrm{ii}}=90\right)\end{array}$} \\
\hline & & $\mathbf{F}$ & $\%$ & $\mathbf{F}$ & $\%$ & $\mathbf{F}$ & $\%$ \\
\hline \multirow[t]{3}{*}{$\mathbf{1}$} & Cultural practices & & & & & & \\
\hline & Alternate drying and wetting & 64 & 71.11 & 54 & 60.00 & 66 & 73.33 \\
\hline & Crop rotation & 73 & 81.11 & 70 & 77.78 & 78 & 86.67 \\
\hline \multirow[t]{4}{*}{2} & Biological practices & & & & & & \\
\hline & Dashaparanikashaya @ 1\% & 65 & 72.22 & $* *$ & $* *$ & $* *$ & $* *$ \\
\hline & Biodigester@10\% & 74 & 82.22 & $* *$ & $* *$ & $* *$ & $* *$ \\
\hline & $\begin{array}{c}\text { Huli majjige (sour butter } \\
\text { milk) @ } 10 \%\end{array}$ & 67 & 74.44 & $* *$ & $* *$ & $* *$ & $* *$ \\
\hline
\end{tabular}

F- Frequency, \%-Per cent, **-Practices not recommended

Since paddy as major crop grown in the study area from many years and majority of the growers had medium to high farming experience (9-16 and more than 17 years) might be the reasons for the above results.

Over half of the organic paddy growers had knowledge about practice of seeds soaking in water $(65.56 \%)$ followed by time of application of compost $(64.44 \%)$ and sucking pests as important pests in nursery $(54.44 \%)$. Majority of them know the benefits of soaking seeds as it increases germination percentage.
Application of compost and sucking pest management are the basic nursery practices, so hence the result.

Knowledge level of inorganic paddy growers about nursery management practices

The results presented in the Table 3 also revealed that cent per cent of inorganic paddy growers had knowledge about seeds required for nursery followed by irrigation management and weed management practices. 
Whereas, a majority of them had knowledge about time of application of recommended NPK $(80.00 \%)$ followed by sucking pests as important pests in nursery (75.56 \%), reddening as first most important disease $(65.56 \%)$ and damping off as second most important disease $(51.11 \%)$.

However, time of application of compost, recommended NPK and recommended compost application was attributed to 43.33, 31.11 and 23.33 per cent, respectively.

These practices are important aspects of nursery management required for raising quality seedlings in cultivation of paddy crop. Therefore, majority of the growers found to have knowledge about these basic practices.

Knowledge level of organic and inorganic paddy growers about nutrient management aspects in main field

Knowledge level of organic paddy growers about nutrient management aspects in main field

It is observed from the Table 4 that, cent per cent of organic paddy growers had knowledge about spraying of panchagavya followed by jeevamrutha and cow urirne. Further, a majority (74.44\%) of them had knowledge about panchagavya preparation and quantity to be sprayed followed by recommended quantity of FYM/compost application and an equal per cent $(72.22 \%)$ with jeevamrutha preparation and quantity to be sprayed.

Whereas, cultivation of Pillipesaru as green manure crops followed by purpose of jeevamrutha spraying as it boosts the plant growth and second time of neem cake application was attributed to 71.11 and 70.00 per cent. However, majority $(68.89 \%)$ of the growers had knowledge about other practices such as purpose of neem cake application as it manages insect pests followed by quantity of neem cake application for second time (64.44 $\%$ ), recommended quantity of vermicompost application $(63.33 \%)$, cultivation of Sunhemp as green leaf manure crop $(61.11 \%)$, purpose of panchagavya spraying as growth promoter $(60.00 \%)$, time of application of neem cake for first time $(57.78 \%)$, quantity of cow urine sprayed $(57.78 \%)$, application of neem cake $(56.67 \%)$, cultivation of green leaf manures (55.56\%), cultivation of Azolla (54.44\%), purpose of application of neem cake as it acts as source of nutrients $(52.22 \%)$, cultivation of Dhaincha as green manure crop (52.22 \%), cultivation of Subabul as green leaf manure crop (51.11 \%), purpose of panchagavya spraying as it increases yield $(51.11 \%)$ and seed rate required for green manure crops accounted for 50.00 per cent.

The possible reasons for better knowledge about nutrient management practices might be due to the fact that, paddy as nutrient exhaustive crop usually requires more of nutrients than other crops, so compost, vermicompost, neem cake, green manure crop and Azolla as solid sources jeevamrutha, panchagavya and cow urine as liquid sources are the only sources of nutrients in organic farming. So this might have motivated growers to know more about nutrient management practices in nursery.

Less than half $(48.89 \%)$ of the organic paddy growers had knowledge about quantity of neem cake application for first time followed by purpose of cow urine spraying as it serve as urea supplement $(47.78 \%)$ and cultivation of Gliricidia as green leaf manure crop (44.44 $\%)$. An equal per cent (34.44\%) of them had knowledge about purpose of cow urine spraying as it has insecticidal property and recommended quantity of poultry manure application. Lowest $(28.89 \%)$ were found in case of quantity of Azolla required for one acre followed by quantity of application of green leaf manure crops $(23.33 \%)$ and 
purpose of pachagavya spraying as it provide immunity in plant system (12.22\%). The probable reason is that, the incidence of pest and disease is generally more in this crop. Hence, growers have better knowledge about paddy cultivation practices and pests and diseases management for getting higher yields. Reasons for the lack of knowledge about the cultivation of green manure crops and Azolla may be low level of education among farmers and lack of technical know how.

\section{Knowledge level of inorganic (transplanting) paddy growers about nutrient management aspects in main field}

It is also observed from the Table 4 that, inorganic paddy growers had knowledge about practices such as application of zinc sulphate and split application (top dressing) of $\mathrm{N}$ fertilizer were found to be cent per cent. Further a majority $(63.33 \%)$ of them had knowledge about recommended compost application followed by recommended $\mathrm{N}$ application (42.22\%), phosphorus application $(38.89 \%)$ and potash application $(32.22 \%)$.

However, the growers had low level of knowledge about iron sulphate application followed by cultivation of Pillipesaru as green manure crop, recommended quantity of vermicompost application, cultivation of green leaf manures, poultry manure application, cultivation of Azolla and Sunhemp were found to be $17.78,10.00,8.89,7.78,6.67,5.56$ and 4.44 per cent, respectively.

It is interesting fact that, none of the inorganic paddy growers had knowledge about cultivation of Dhaincha as green manure followed by seed rate of green manure crops required per acre, cultivation of Gliricidia and Subabul as green leaf manure, application quantity of green leaf manure for one acre and quantity of Azolla required for one acre. This might be due to lack of awareness and technical know how among the growers regarding the importance of these practices.

Knowledge level of inorganic (DSR) paddy growers about nutrient management aspects in main field

It is observed from the Table 4 that, cent per cent of the inorganic paddy growers had knowledge about practices such as application of zinc sulphate and split application (top dressing) of $\mathrm{N}$ fertilizer. The probable reason might be that all the paddy growers gain importance of split application of nitrogenous fertilizers and micro nutrient application (Zinc sulphate).

Further 63.33 per cent of them had knowledge about recommended compost application followed by recommended $\mathrm{N}$ application (57.78 \%), phosphorus application (52.22\%), potash application (45.56\%) and iron sulphate application $(23.33 \%)$, respectively. As these are the basic cultivation practices known by every growers.

Knowledge level on traditional practices by organic and inorganic paddy growers about plant protection measures

Knowledge level on traditional practices by organic paddy growers about plant protection measures

The close persual from the Table 5 that, majority $(82.22 \%)$ of the growers had knowledge about usage of biodigester followed by crop rotation $(81.11 \%)$ spraying of huli majjige (74.44 \%), spraying of Dhashaparani kashaya (72.22 \%) and practice of alternate drying and wetting $(71.11 \%)$.

The possible reasons for better knowledge about pest management practices might be due to the fact that, paddy is more sensitive crop and is prone to many pest and diseases. So 
management of pest and diseases is important for successive cultivation and higher yields and also non application of chemicals might have motivated the growers to know about these practices. The technical guidance provided by the Department of Agriculture and NGos working in the area might also be the one reason for above results.

Knowledge level on traditional practices by inorganic (transplanting) paddy growers about plant protection measures

The findings from the Table 5 also conveys that, majority $(77.78 \%)$ of the growers had knowledge about crop rotation followed by practice of alternate drying and wetting $(60.00$ $\%)$. Experience of growers in paddy cultivation and importance of these practices in pest and disease management might be the probable reason for the above results.

Knowledge level on traditional practices by inorganic (DSR) paddy growers about plant protection measures

The findings from the Table 5 also conveys that, majority $(86.67 \%)$ of the growers had knowledge about crop rotation followed by practice of alternate drying and wetting (73.33 $\%)$. Experience of growers in paddy cultivation and importance of these practices in pest and disease management might be the probable reason for the above results.

\section{References}

Sihare, A. 2015. A study on farmers knowledge, attitude and practices related to organic farming in Tikamgarh district of Madhya Pradesh. M. Sc. (Agri.) Thesis, Jawaharlal Nehru Krishi Vishwa Vidyalaya, Jabalpur.

Motlhanka, K., Maraddi, G. N., Meti, S. K. and Anand, N. 2019. Knowledge domain analysis of ecofriendly direct seeded rice versus transplanted rice cultivation in North east Karnataka. Journal of farm Science, 32 (2), 201206.

Naik, M. H., Srivastava, S. R., Godara, A. K. and Yadav, V. P. S. 2009. Knowledge level about organic farming in Haryana. Indian Research Jornal of Extension Education, 9 (1), 50-53.

Sharanappa, G. T. 2011. A study on knowledge and adoption of recommended production practices of paddy by the farmers of Tugabhadra Project Area, Karnataka. M. Sc (Agri.) Thesis, University of Agricultural Sciences, Raichur.

\section{How to cite this article:}

Sidramayya, S. K. Meti, D. M. Chandargi and Satyanarayana Rao. 2019. Knowledge Level of Organic and Inorganic Paddy Growers about Cultivation Practices in TBP Command Area. Int.J.Curr.Microbiol.App.Sci. 8(12): 3056-3069. doi: https://doi.org/10.20546/ijcmas.2019.812.356 